\title{
Synthesis and isomerization of enyne-group containing compounds in NaEDA/EDA media
}

\author{
Artur Jõgi and Uno Mäeorg* \\ Institute of Organic Chemistry, University of Tartu, Jakobi 2, Tartu, 51014, Estonia \\ E-mail: uno@chem.ut.ee
}

\begin{abstract}
Dedicated to Professor Kalevi Pihlaja on the occasion of his $60^{\text {th }}$ birthday
(received 20 Apr 01; accepted 01 Jan 99; published on the web 11 Mar 02)
\end{abstract}

\begin{abstract}
A series of conjugated enyne-group and isolated triple bond containing compounds were synthesized and their behavior in superbasic media NaEDA/EDA studied.
\end{abstract}

Keywords: Conjugated enynes, isomerization, superbases

\section{Introduction}

The multipositional migration of the triple bond along the carbon chain in hydrocarbons and alcohols by potassium 3 -aminopropanamide was introduced by Brown ${ }^{1}$. Later other superbasic systems based on different alkali metal amides of 1,2-diaminoethane and 1,3-diaminopropane for such processes were developed ${ }^{2-5}$. The triple bond usually migrates to the terminal position of carbon chain but in branched chain compounds ${ }^{6}$ and carboxylic acids ${ }^{7,8}$, several other compounds have been obtained. Remissive ET al. ${ }^{9,10}$ have described the successful migration of conjugated diyne group in hydrocarbons and alcohols with LiEDA/EDA. The conjugated enyne group was still isomerized only at 1-position in (Z, E)-2-hexen-3-yn-1-ol by influence of $\mathrm{KNH}_{2} / \mathrm{NH}_{3}{ }^{11}$. The lack of the data on multipositional isomerization of enyne-group inspired us to investigate this type of reaction on different model compounds to show the scope and limitations of this transformation.

\section{Results and Discussion}

Recently, we have described a successful isomerization of (Z, E)-2-octen-4-yn-1-ol (1) to (Z, E)5-octen-7-yn-1-ol (8) by NaEDA/EDA ${ }^{12}$. Here, we extend this study to other possible enynic substrates. The present results (cf. Table) indicate that the presence of functional groups in conjugated enynes influence substantially the isomerization. For example, (Z, E)-2-octen-4-yn-1- 
ol (1) $(Z / E=33 / 67)$ isomerizes by the influence of NaEDA/EDA to $(Z, E)-5$-octen-7-yn-1-ol (8) $(Z / E=65 / 35)$ with high yield. On the other hand, the isomerization product of $(Z, E)-4$-undecen6-yne (2) $(Z / E=66 / 34)$ was contaminated with substantial amount of polymeric side products. After chromatographic separation on silica column, the $(Z, E)-3$-undecen-1-yne (9) $(Z / E=52 / 48)$ was obtained with $44 \%$ yield. The change of the hydroxy group in compound (3) $(Z / E=50 / 50)$ to butoxy group led even at the mildest reaction conditions (LiEDA/EDA) only to the polymeric product.

It appears that the order of double and triple bond with respect of the functional group in enynol also influences the isomerization. Thus, the position of enyne group in (Z, E)-4-octen-2yn-1-ol (4) $(Z / E=56 / 44)$ remained unchanged and only $Z-E$ isomerization $(10)(Z / E=50 / 50)$ was observed in NaEDA/EDA.

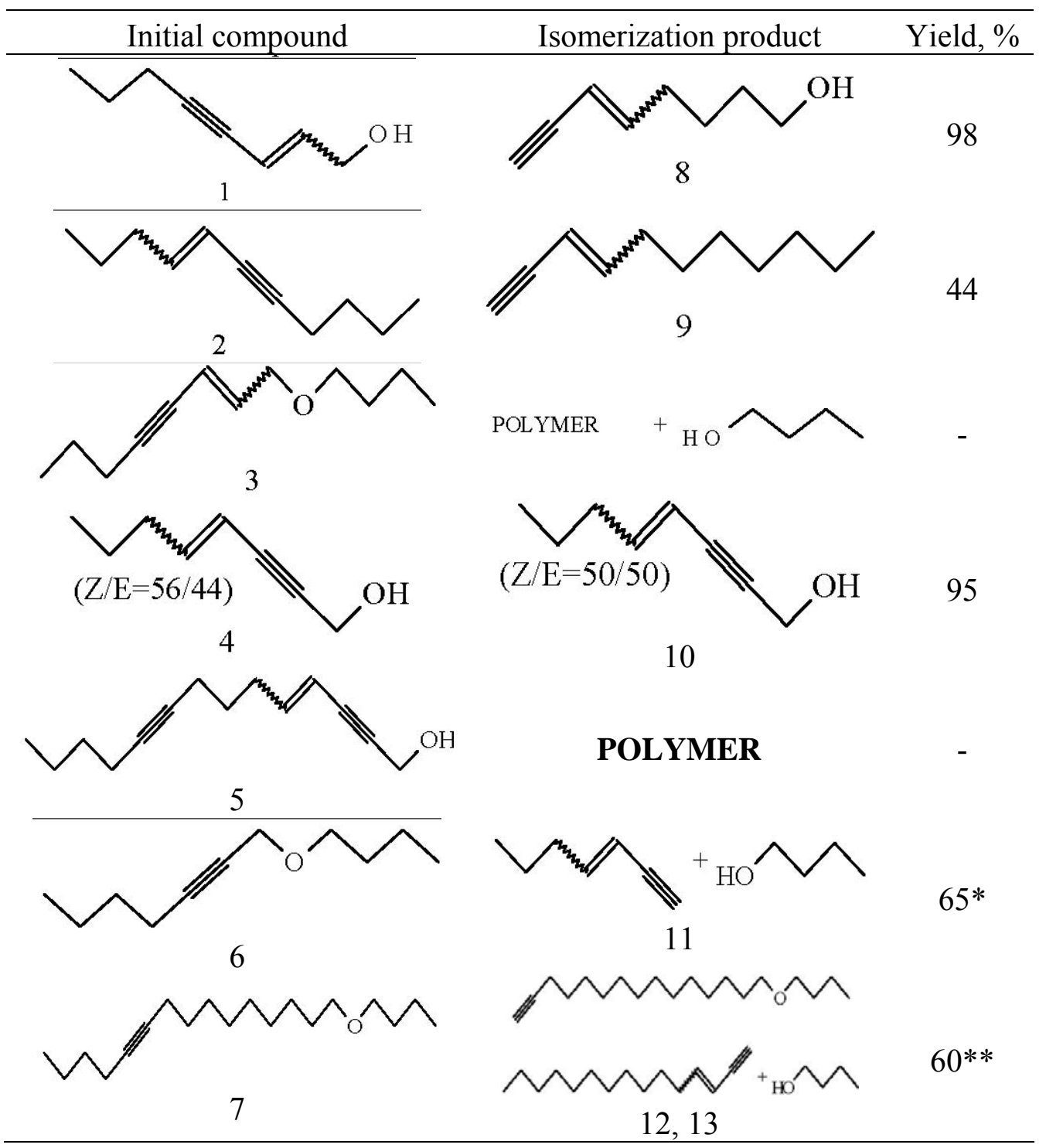

* Total yield, Compound (11)/1-butanol=1/1.

** Total yield, $(\mathbf{1 2}) /(\mathbf{1 3})=1 / 1,(\mathbf{1 3}) / 1-$ butanol=1/1. 
By using this knowledge, we synthesized the compound (5) $(Z / E=47 / 53)$ to test the stability of the enynol group and the reactivity of the isolated triple bond in its neighborhood. Unfortunately the product polymerized even using LiEDA/EDA.

Due to the polymerization of 1-butoxy-(Z,E)-2-octen-4-yne (3) $(Z / E=50 / 50)$, the behavior of simple propargylic ether in NaEDA/EDA was tested. Such ethers are known as starting materials for the preparation of enynes by influence of $\mathrm{NaNH}_{2} / \mathrm{NH}_{3}{ }^{13}$. In our experiment with NaEDA/EDA, the corresponding (Z, E)-3-hepten-1-yne (11) was obtained with good yield ( $Z$ : $E=1: 2$ ). In order to study the influence of the position of the triple bond with respect of the alloy group, we synthesized 1-butoxy-10-pentadecyne (7). By influence of NaEDA/EDA, two products containing triple bond were obtained as the $1: 1$ mixture with total yield of $60 \%$. The mixture was separated by column chromatography on silica. One of these products was the terminal enyne $(\mathrm{Z} / \mathrm{E}=50 / 50)$ whereas the other was determined as the butoxy derivative with terminal triple bond. According to those results, the triple bond can migrate to the both ends of carbon chain because several positions may be deprotonated and in equilibrium with each other. When the triple bond moves to the alkoxy group, the butanol is eliminated and after the isomerization the compound with the terminal enyne is formed ${ }^{13}$. Another terminal position for the triple bond migration can be localized at the other end of molecule because of higher acidity of the acetylenic proton.

It has to be concluded that $\mathrm{Na}$ and LiEDA/EDA are powerful superbases for the multipositional isomerization of enyne-group in hydrocarbons and alcohols. These superbases are though too strong in the case of some compounds and cause polymerization.

\section{Experimental Section}

General Procedures. ${ }^{1} \mathrm{H}$ and ${ }^{13} \mathrm{C}$ NMR spectra were measured with Bruker AC 200P (Spektrospin AG, Switzerland) spectrometer at 200 and $50 \mathrm{MHz}$, respectively. IR spectra were recorded on Specord M82 Spectrometer (Carl Zeiss, Jena). GLC analyses: Chrom-5 (Laboratorni Pristroje, Praha), glass columns: $2.5 * 3 \mathrm{~mm}, 20 \%$ Carbowax 20M, Chromosorb W/AW-DMCS, 100-120 mesh; 5\% PDEAS, Chromosorb W/HP AW-DMCS, 100-120 mesh; and fused silica capillary column, $0.33 * 24 \mathrm{~m}$, SGE, BP10. For thin layer chromatography Silufol $\mathrm{UV}_{254}$ (Chavalier) plates with silica gel were used. Preparative chromatography was carried out on columns packed with 40-63 $\mu \mathrm{m}$ silica gel. Reagents and chemicals were obtained from Aldrich Chemical Company (USA) and from REACHIM (USSR).

All isomerization experiments were done under dry and oxygen-free Ar-atmosphere. Ar was passed through the column $(2.5 * 23 \mathrm{~cm})$ with $\mathrm{Ni}\left(\mathrm{CrO}_{2}\right)_{2}$ granulated catalyst as oxygen bonding agent and through the column $(4 * 18 \mathrm{~cm})$ with molecular sieves $4 \AA$ to remove $\mathrm{H}_{2} \mathrm{O}$ and $\mathrm{CO}_{2}$. The batch of ethylenediamine (EDA) was distilled first from $\mathrm{KOH}$ and then two times above $\mathrm{Na}$ under Ar-atmosphere. For every experiment EDA was distilled freshly from Na under argon. 
General Procedures. To a dry stirred EDA (3 mL) under Ar-atmosphere $\mathrm{NaNH}_{2}(0.195 \mathrm{~g}, 0.005$ mol) was added and the mixture was stirred at room temperature until the gray colour changed to violet $(0.5 \mathrm{~h})$. The compound studied $(0.001 \mathrm{~mol})$ was added to $1.7 \mathrm{M} \mathrm{NaEDA} / \mathrm{EDA}$ solution. The stirring was continued and the reaction was monitored by GLC. When reaction was over, the mixture was hydrolyzed by ice water, extracted with ether and washed first with the $10 \% \mathrm{HCl}$ solution, then with the saturated solutions of $\mathrm{NaHCO}_{3}$ and $\mathrm{NaCl}$. The organic phases were dried over $\mathrm{MgSO}_{4}$ and concentrated in vacuo. The remaining brown liquid was purified using column chromatography on silica.

LiEDA/EDA (1.2M solution) was obtained by the reaction of EDA with Li $(0.042 \mathrm{~g}, 0.006 \mathrm{~mol})$ during 0.5 hour until the colour of the mixture changed from blue to white.

(Z,E)-2-Octen-4-yn-1-ol (1). Described in ref. 12.

(Z, E)-4-Undecen-6-yne (2). Prepared analogously to the published method ${ }^{15}$ by the following scheme:

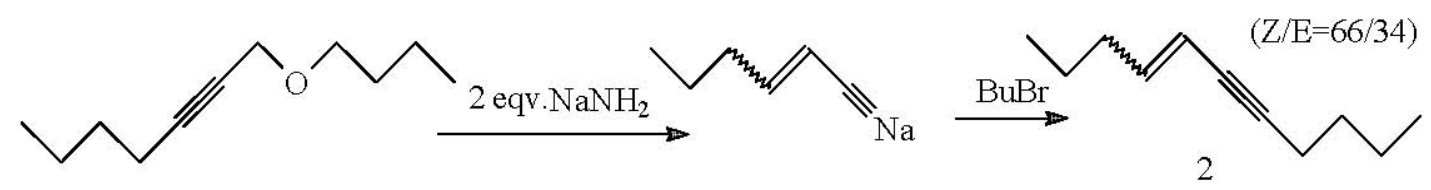

${ }^{1} \mathrm{H}$ NMR $\left(\mathrm{CDCl}_{3}\right) \delta$ 6.15-5.75 $\left(1 \mathrm{H}, \mathrm{m},-\mathrm{CH}_{2}-\mathrm{CH}=\right)$, 5.52-5.36 $(1 \mathrm{H}, \mathrm{m},=\mathrm{CH}-\mathrm{C} \equiv), 2.20-1.98(4 \mathrm{H}$, $\left.\mathrm{m},-\mathrm{CH}_{2}-\mathrm{C} \equiv,-\mathrm{CH}_{2}-\mathrm{CH}=\right), 1.6-1.3\left(6 \mathrm{H}, \mathrm{m}, 3 \mathrm{x}-\mathrm{CH}_{2}-\right), 0.98-0.82\left(6 \mathrm{H}, \mathrm{m}, 2 \mathrm{x} \mathrm{CH} 3^{-}\right) ;{ }^{13} \mathrm{C}$ NMR $\left(\mathrm{CHCl}_{3}\right) \delta 142.9\left(4-\mathrm{C}_{\mathrm{E}}\right), 142.1\left(4-\mathrm{C}_{\mathrm{Z}}\right), 110.3\left(5-\mathrm{C}_{\mathrm{E}}\right), 109.8\left(5-\mathrm{C}_{\mathrm{Z}}\right), 94.38\left(7-\mathrm{C}_{\mathrm{E}}\right), 88.62\left(7-\mathrm{C}_{\mathrm{Z}}\right)$, $79.34\left(6-C_{E}\right), 77.56\left(6-C_{Z}\right), 35.11\left(3-C_{E}\right), 32.17\left(3-C_{Z}\right), 31.16(9-C), 22.30\left(2-C_{Z}\right), 22.19\left(2-C_{E}\right)$, $22.09\left(8-\mathrm{C}_{\mathrm{E}}\right), 22.05\left(8-\mathrm{C}_{\mathrm{Z}}\right), 19.31\left(10-\mathrm{C}_{\mathrm{Z}}\right), 19.15\left(10-\mathrm{C}_{\mathrm{E}}\right), 13.75(1-\mathrm{C}), 13.62(11-\mathrm{C}) ; \mathrm{IR}(\mathrm{KBr})$ $3024,1660,956,733 \mathrm{~cm}^{-1}$.

(Z, E)-3-Undecen-1-yne (9). ${ }^{1} \mathrm{H}$ NMR $\left(\mathrm{CDCl}_{3}\right) \delta$ 6.15-5.90 $\left(1 \mathrm{H}, \mathrm{m},=\mathrm{CH}-\mathrm{CH}_{2}-\right), 5.55-5.35(1 \mathrm{H}$, $\mathrm{m}, \equiv \mathrm{C}-\mathrm{CH}=), 3.06 / 2.76(1 \mathrm{H}, 2 \mathrm{x} \mathrm{d}, \mathrm{HC} \equiv$, cis/trans $), 2.40-1.95\left(2 \mathrm{H}, \mathrm{m},-\mathrm{CH}_{2}-\mathrm{CH}=\right), 1.55-1.10$ $\left(10 \mathrm{H}, \mathrm{m}, 5 \mathrm{x}-\mathrm{CH}_{2}-\right), 0.93\left(3 \mathrm{H}, \mathrm{t}, \mathrm{CH}_{3^{-}}\right) ;{ }^{13} \mathrm{C} \mathrm{NMR}\left(\mathrm{CHCl}_{3}\right) \delta 146.9\left(4-\mathrm{C}_{\mathrm{E}}\right), 146.1\left(4-\mathrm{C}_{\mathrm{Z}}\right), 108.6$ $\left(3-C_{E}\right), 108.1\left(3-C_{Z}\right), 81.12\left(1-C_{E}\right), 82.66\left(2-C_{Z}\right), 80.66\left(2-C_{E}\right), 75.49\left(1-C_{Z}\right), 33.03\left(5-C_{E}\right), 31.83$ (5-C $\mathrm{Z}_{\mathrm{Z}}$ ), 29.76 (6-C, 7-C, 8-C), 29.12 (9-C), 22.68 (10-C), 14.06 (11-C); IR (KBr) 3320, 3024, $2110,1656,962,764,641 \mathrm{~cm}^{-1}$.

1-Butoxy- (Z, E)-2-octen-4-yne (3). Obtained by the scheme ${ }^{14}$ :

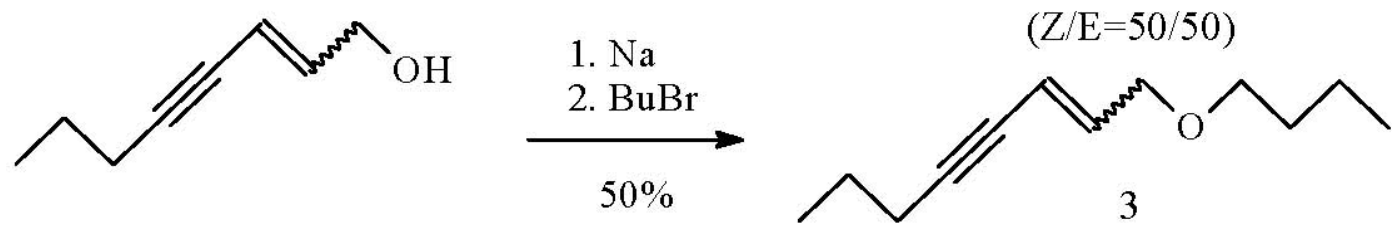

${ }^{1} \mathrm{H}$ NMR $\left(\mathrm{CDCl}_{3}\right) \delta$ 6.15-5.55 $\left(2 \mathrm{H}, \mathrm{m},=\mathrm{CH}-\mathrm{CH}_{2^{-}}, \equiv \mathrm{C}-\mathrm{CH}=\right), 4.22 / 3.97\left(2 \mathrm{H}, 2 \mathrm{x} \mathrm{d},=\mathrm{CH}-\mathrm{CH}_{2^{-}}\right.$, cis/trans), 3.55-3.35 (2H, m, -O- $\left.\mathrm{CH}_{2}-\mathrm{CH}_{2}-\right), 2.35-2.20\left(2 \mathrm{H}, \mathrm{m},-\mathrm{CH}_{2}-\mathrm{C} \equiv\right)$, 1.7-1.2 (6H, m, 3x $\left.\mathrm{CH}_{2^{-}}\right), 1.10-0.82\left(6 \mathrm{H}, \mathrm{m}, 2 \mathrm{x} \mathrm{CH} 3^{-}\right) ;{ }^{13} \mathrm{C} \mathrm{NMR}\left(\mathrm{CHCl}_{3}\right) \delta 138.4\left(2-\mathrm{C}_{\mathrm{E}}\right), 138.3\left(2-\mathrm{C}_{\mathrm{Z}}\right), 112.2$ (3- 
$\left.\mathrm{C}_{\mathrm{E}}\right), 111.8\left(3-\mathrm{C}_{Z}\right), 96.19\left(5-\mathrm{C}_{\mathrm{E}}\right), 90.77\left(5-\mathrm{C}_{\mathrm{Z}}\right), 78.81\left(4-\mathrm{C}_{\mathrm{E}}\right), 76.80\left(4-\mathrm{C}_{\mathrm{Z}}\right), 70.68\left(1-\mathrm{C}_{\mathrm{E}}\right), 70.35$ $\left(1-C_{Z}\right), 68.48\left(1^{`}-C\right), 31.98\left(2^{`}-C\right), 22.29$ (7-C), $21.61\left(6-C_{Z}\right), 21.47\left(6-C_{E}\right), 19.44\left(3^{`}-C\right), 13.90$ (4'-C), 13.48 (8-C); IR (KBr) 3024, 1658, 1103, 974, $764 \mathrm{~cm}^{-1}$.

(Z, E)-4-Octen-2-yn-1-ol (4). Prepared according to the literature ${ }^{11}$. Bp: $118-121^{\circ} \mathrm{C} / 5-6 \mathrm{mmHg}$. ${ }^{1} \mathrm{H}$ NMR $\left(\mathrm{CDCl}_{3}\right) \delta$ 6.25-5.85 $\left(1 \mathrm{H}, \mathrm{m},=\mathrm{CH}-\mathrm{CH}_{2}-\right), 5.55-5.45(1 \mathrm{H}, \mathrm{m}, \equiv \mathrm{C}-\mathrm{CH}=), 4.41 / 4.36(2 \mathrm{H}$, 2x s, $-\mathrm{CH}_{2}-\mathrm{O}-$, cis/trans), $2.76(1 \mathrm{H}, \mathrm{s},-\mathrm{OH}), 2.35-2.0\left(2 \mathrm{H}, \mathrm{m}, \mathrm{CH}_{3}-\mathrm{CH}_{2}-\mathrm{CH}_{2}-\right), 1.55-1.30(2 \mathrm{H}, \mathrm{m}$, $\left.\mathrm{CH}_{3}-\mathrm{CH}_{2}-\right), 1.0-0.85\left(3 \mathrm{H}, \mathrm{m}, \mathrm{CH}_{3}-\right) ;{ }^{13} \mathrm{C} \mathrm{NMR}\left(\mathrm{CHCl}_{3}\right) \delta 145.3\left(5-\mathrm{C}_{\mathrm{E}}\right), 144.4\left(5-\mathrm{C}_{\mathrm{Z}}\right), 109.2$ (4$\left.\mathrm{C}_{\mathrm{E}}\right), 108.6\left(4-\mathrm{C}_{Z}\right), 91.51\left(3-\mathrm{C}_{Z}\right), 85.89\left(3-\mathrm{C}_{\mathrm{E}}\right), 84.55\left(2-\mathrm{C}_{\mathrm{E}}\right), 82.59\left(2-\mathrm{C}_{\mathrm{Z}}\right), 51.58\left(1-\mathrm{C}_{\mathrm{Z}}\right), 51.48$ $\left(1-C_{E}\right), 35.12\left(6-C_{E}\right), 32.32\left(6-C_{Z}\right), 22.13\left(7-C_{Z}\right), 21.95\left(7-C_{E}\right), 13.88\left(8-C_{Z}\right), 13.55\left(8-C_{E}\right)$; IR (KBr) 3321, 3018, 1660, 1011, 956, 733, $616 \mathrm{~cm}^{-1}$.

(Z, E)-4-Tetradecen-2.9-diyn-1-ol (5). Prepared according to the literature ${ }^{11}$ in a similar way by the following scheme:
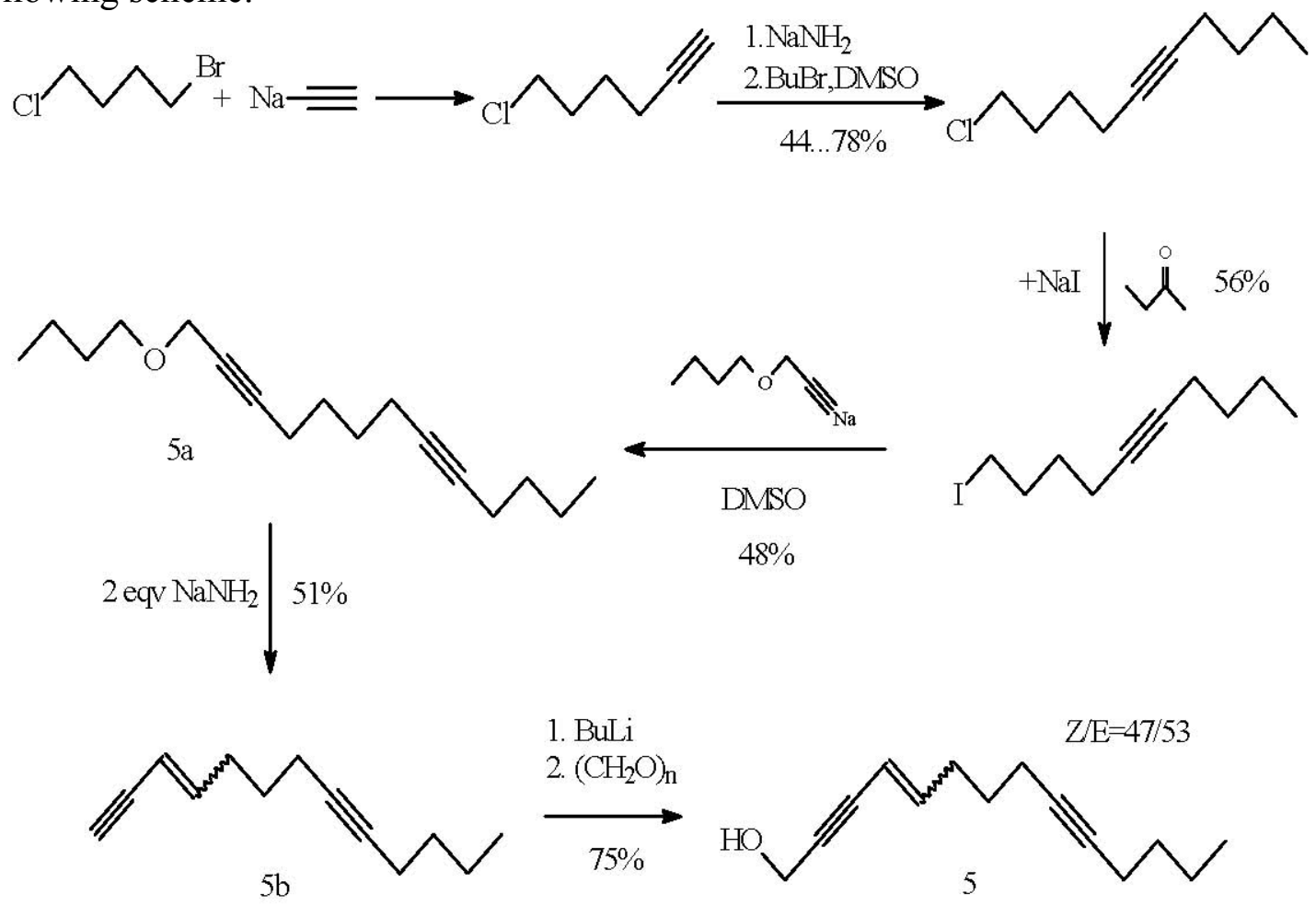

Intermediates and final products were purified by column chromatography $($ EtAc/Hexane $=1 / 3)$ and characterized by NMR and IR spectra:

1-Butoxy-2.8-tridecadiyne (5a). ${ }^{1} \mathrm{H} \mathrm{NMR}\left(\mathrm{CDCl}_{3}\right) \delta 4.09\left(2 \mathrm{H}, \mathrm{t}, \mathrm{J}=2.2,-\mathrm{O}-\mathrm{CH}_{2}-\mathrm{C} \equiv\right), 3.48(2 \mathrm{H}$, $\left.\mathrm{t}, \mathrm{J}=6.6,-\mathrm{CH}_{2}-\mathrm{CH}_{2}-\mathrm{O}-\right), 2.30-2.08\left(6 \mathrm{H}, \mathrm{m},-\mathrm{CH}_{2}-\right), 1.65-1.52\left(2 \mathrm{H}, \mathrm{m},-\mathrm{CH}_{2}-\right), 1.48-1.32(10 \mathrm{H}, \mathrm{m}$,

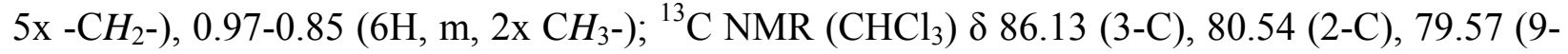
C), 76.74 (8-C), 69.65 (1`-C), 58.57 (1-C), 31.81 (2`-C), 31.42 (11-C), 28.39 (5-C), 27.91 (6-C), 22.03 (12-C), 19.46 (4-C), 18.52 (7-C), 18.45 (10-C), 18.41 (3`-C), 13.88 (4 -C), 13.62 (13-C); IR (KBr) $1141,1098 \mathrm{~cm}^{-1}$.

(Z, E)-3-Tridecen-1.8-diyne (5b). ${ }^{1} \mathrm{H}$ NMR $\left(\mathrm{CDCl}_{3}\right) \delta$ 6.32-5.92 (1H, m, $\left.=\mathrm{CH}-\mathrm{CH}_{2}-\right)$, 5.55-5.40 
$(1 \mathrm{H}, \mathrm{m}, \equiv \mathrm{C}-\mathrm{CH}=), 3.08 / 2.78\left(1 \mathrm{H}, 2 \mathrm{x} \mathrm{d}, \mathrm{HC} \equiv\right.$, cis/trans), 2.55-2.05 $\left(6 \mathrm{H}, \mathrm{m}, 3 \mathrm{x}-\mathrm{CH}_{2}-\right), 1.7-1.2$ $\left(6 \mathrm{H}, \mathrm{m}, 3 \mathrm{x}-\mathrm{CH}_{2-}\right), 1.02-0.80\left(3 \mathrm{H}, \mathrm{m}, \mathrm{CH}_{3^{-}}\right) ;{ }^{13} \mathrm{C} \mathrm{NMR}\left(\mathrm{CHCl}_{3}\right) \delta 145.7\left(4-\mathrm{C}_{\mathrm{E}}\right), 144.9\left(4-\mathrm{C}_{\mathrm{Z}}\right)$, $109.4\left(3-C_{E}\right), 108.8\left(3-C_{Z}\right), 82.46\left(2-C_{Z}\right), 81.51\left(1-C_{E}\right), 80.98\left(2-C_{E}\right), 80.73\left(8-C_{E}\right), 80.39\left(8-C_{Z}\right)$, $79.52\left(9-C_{Z}\right), 79.25\left(9-C_{E}\right), 75.84\left(1-C_{Z}\right), 32.02\left(11-C_{E}\right), 31.33\left(11-C_{Z}\right), 29.76\left(5-C_{E}\right), 29.54(5-$ $\left.\mathrm{C}_{\mathrm{Z}}\right), 28.46$ (6-C $\left.\mathrm{E}\right), 28.08\left(6-\mathrm{C}_{\mathrm{Z}}\right), 21.99$ (12-C), 18.50 (7-C), 18.24 (10-C), 13.61 (13-C); IR (KBr) $3308,3024,2110,1656,962,746,635 \mathrm{~cm}^{-1}$.

(Z, E)-4-Tetradecen-2.9-diyn-1-ol (5). ${ }^{1} \mathrm{H}$ NMR $\left(\mathrm{CDCl}_{3}\right) \delta$ 6.22-5.83 (1H, m, =CH-CH- - $)$, 5.6$5.45(1 \mathrm{H}, \mathrm{m}, \equiv \mathrm{C}-\mathrm{CH}=), 4.40 / 4.35\left(2 \mathrm{H}, 2 \mathrm{x} \mathrm{s},-\mathrm{O}-\mathrm{CH}_{2}-\mathrm{C} \equiv\right.$, cis/trans $), 3.20$ (1H, s, HO-), 2.50-2.05 $\left(6 \mathrm{H}, \mathrm{m}, 3 \mathrm{x}-\mathrm{CH}_{2^{-}}\right), 1.70-1.23\left(6 \mathrm{H}, \mathrm{m}, 3 \mathrm{x}-\mathrm{CH}_{2^{-}}\right), 1.02-0.82\left(3 \mathrm{H}, \mathrm{m}, \mathrm{CH}_{3^{-}}\right) ;{ }^{13} \mathrm{C} \mathrm{NMR}\left(\mathrm{CHCl}_{3}\right) \delta$ $144.3\left(5-C_{E}\right), 143.4\left(5-C_{Z}\right), 109.8\left(4-C_{E}\right), 109.3\left(4-C_{Z}\right), 92.00\left(3-C_{Z}\right), 86.32\left(2-C_{E}\right), 84.22\left(3-C_{E}\right)$, $82.22\left(9-C_{E}\right), 81.00\left(10-C_{Z}\right), 80.77\left(9-C_{Z}\right), 79.77\left(5-C_{E}\right), 79.38\left(2-C_{Z}\right), 51.39\left(1-C_{E}\right), 51.31$ (1$\left.\mathrm{C}_{\mathrm{Z}}\right), 32.06\left(12-\mathrm{C}_{\mathrm{E}}\right), 31.34\left(12-\mathrm{C}_{\mathrm{Z}}\right), 29.52\left(6-\mathrm{C}_{\mathrm{E}}\right), 28.55\left(6-\mathrm{C}_{\mathrm{Z}}\right), 28.21$ (7-C), 22.00 (13-C), 18.49 (8-C), 18.24 (11-C), 13.61 (14-C); IR (KBr) 3364, 3024, 1660, 1017, 962, 740, $628 \mathrm{~cm}^{-1}$.

1-Butoxy-2-heptyne (6). Prepared from propargyl alcohol according to the literature ${ }^{11}$. Bp: 75 $80{ }^{\circ} \mathrm{C} / 3-4 \mathrm{mmHg}$.

(Z, E)-3-Hepten-1-yne (11). ${ }^{1} \mathrm{H}$ NMR $\left(\mathrm{CDCl}_{3}\right) \delta$ 6.35-5.90 $\left(1 \mathrm{H}, \mathrm{m},=\mathrm{CH}_{-} \mathrm{CH}_{2}-\right), 5.53-5.38(1 \mathrm{H}$, $\mathrm{m}, \equiv \mathrm{C}-\mathrm{CH}=), 3.06 / 2.76(1 \mathrm{H}, 2 \mathrm{x} \mathrm{d}, \mathrm{HC} \equiv$, cis/trans $), 2.4-2.0\left(2 \mathrm{H}, \mathrm{m},-\mathrm{CH}_{2}-\mathrm{CH}=\right), 1.55-1.30(2 \mathrm{H}$, m, $\left.-\mathrm{CH}_{2^{-}}\right), 0.93\left(3 \mathrm{H}, \mathrm{t}, \mathrm{CH}_{3^{-}}\right) ;{ }^{13} \mathrm{C} \mathrm{NMR}\left(\mathrm{CHCl}_{3}\right) \delta 146.5\left(4-\mathrm{C}_{\mathrm{E}}\right), 145.8\left(4-\mathrm{C}_{\mathrm{Z}}\right), 108.9\left(3-\mathrm{C}_{\mathrm{E}}\right)$, $108.4\left(3-C_{Z}\right), 82.66\left(2-C_{E}\right), 81.20\left(1-C_{E}\right), 80.66\left(2-C_{E}\right), 75.59\left(1-C_{Z}\right), 35.14\left(5-C_{E}\right), 32.35\left(5-C_{Z}\right)$, $22.14\left(6-C_{E}\right), 21.92\left(6-C_{Z}\right), 13.68\left(7-C_{Z}\right), 13.57\left(7-C_{E}\right)$; IR (KBr) 3302, 3024, 2104, 1660, 962, $616 \mathrm{~cm}^{-1}$.

15-Butoxy-5-pentadecyne (7). Prepared according to the literature ${ }^{14}$ by following scheme:

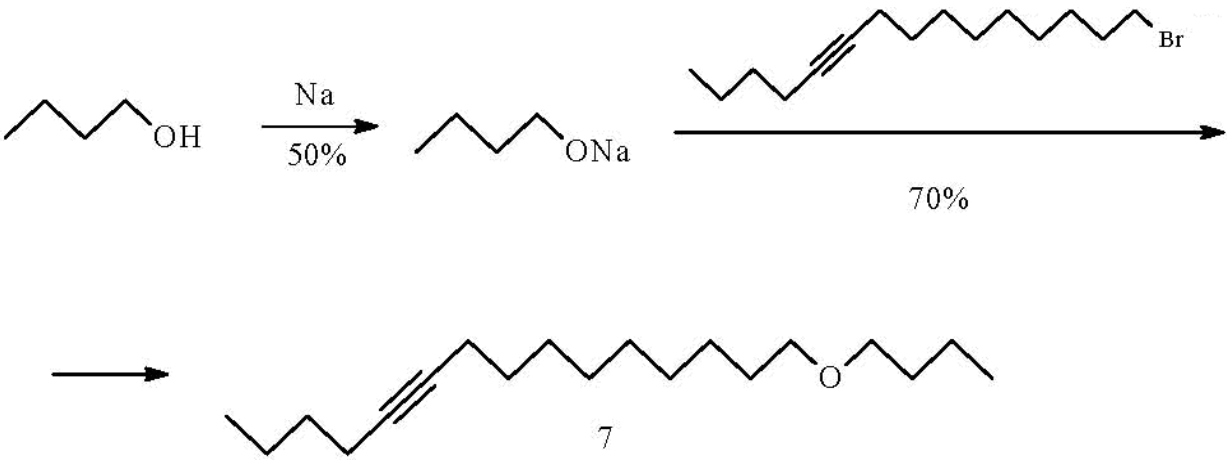

${ }^{1} \mathrm{H}$ NMR $\left(\mathrm{CDCl}_{3}\right) \delta 3.40 / 3.39\left(4 \mathrm{H}, 2 \mathrm{x} \mathrm{t},-\mathrm{CH}_{2}-\mathrm{O}-\mathrm{CH}_{2}\right.$, , cis/trans), 2.22-2.08 (4H, m, $2 \mathrm{x}-\mathrm{CH}_{2}-$ $\mathrm{C} \equiv), 1.65-1.22\left(22 \mathrm{H}, \mathrm{m}, 11 \mathrm{x}-\mathrm{CH}_{2^{-}}\right), 0.98-0.85\left(6 \mathrm{H}, \mathrm{m}, 2 \mathrm{x} \mathrm{CH} 3^{-}\right) ;{ }^{13} \mathrm{C} \mathrm{NMR}\left(\mathrm{CHCl}_{3}\right) \delta 80.18(5-$ C, 6-C), 71.02 (1`-C), 70.70 (15-C), 32.04 (2`-C), 31.42 (3-C), 29.92 (11-C), 29.58 (12-C), 29.54 (9-C), 29.30 (10-C), 29.19 (8-C), 28.92 (14-C), 26.32 (13-C), 21.99 (2-C), 19.49 (3`-C), 18.84 (7-C), 18.53 (4-C), 13.92 (4 -C), 13.61 (1-C); IR (KBr) $1092 \mathrm{~cm}^{-1}$.

15-Butoxy-1-pentadecyne (12). ${ }^{1} \mathrm{H}$ NMR $\left(\mathrm{CDCl}_{3}\right) \delta 3.40 / 3.39\left(4 \mathrm{H}, 2 \mathrm{x}\right.$ t, $-\mathrm{CH}_{2}-\mathrm{O}-\mathrm{CH}_{2-}$, cis/trans), 2.23-2.12 (4H, m, 2x - $\left.\mathrm{CH}_{2}-\mathrm{C} \equiv\right), 1.93(1 \mathrm{H}, \mathrm{t}, \mathrm{J}=2.4, \mathrm{HC} \equiv), 1.63-1.18(26 \mathrm{H}, \mathrm{m}, 11 \mathrm{x}-$ $\left.\mathrm{CH}_{2-}\right), 0.92\left(3 \mathrm{H}, \mathrm{t}, \mathrm{J}=7.2, \mathrm{CH}_{3^{-}}\right) ;{ }^{13} \mathrm{C} \mathrm{NMR}\left(\mathrm{CHCl}_{3}\right) \delta 85.68$ (2-C), 71.95 (1 $\left.{ }^{-}-\mathrm{C}\right), 71.61$ (15-C), 
68.98 (1-C), 32.91 (2`-C), 30.81 (7-C), 30.57 (9-C, 10-C, 11-C, 12-C), 30.48 (14-C, 8-C), 30.09 (6-C), 29.75 (4-C), 29.52 (5-C), 27.21 (13-C), 20.37 (3`-C), 19.39 (3-C), 14.86 (4`-C); IR (KBr) $3315,1116 \mathrm{~cm}^{-1}$.

(Z,E)-3-Pentadecen-1-yne (13). ${ }^{1} \mathrm{H}$ NMR $\left(\mathrm{CDCl}_{3}\right) \delta$ 6.23-5.90 (1H, m, $\left.=\mathrm{CH}-\mathrm{CH}_{2}-\right)$, 5.55-5.35 $(1 \mathrm{H}, \mathrm{m}, \equiv \mathrm{C}-\mathrm{CH}=), 3.06 / 2.76(1 \mathrm{H}, 2 \mathrm{x} \mathrm{d}, \mathrm{HC} \equiv$, cis/trans $), 2.4-2.0\left(2 \mathrm{H}, \mathrm{m},-\mathrm{CH}_{2}-\mathrm{CH}=\right), 1.55-1.05$ $\left(18 \mathrm{H}, \mathrm{m}, 9 \mathrm{x}-\mathrm{CH}_{2-}\right), 0.91\left(3 \mathrm{H}, \mathrm{t}, \mathrm{CH}_{3^{-}}\right) ;{ }^{13} \mathrm{C} \mathrm{NMR}\left(\mathrm{CHCl}_{3}\right) \delta 146.8\left(4-\mathrm{C}_{\mathrm{E}}\right), 146.1\left(4-\mathrm{C}_{\mathrm{Z}}\right), 108.6$ $\left(3-C_{E}\right), 108.1\left(3-C_{Z}\right), 82.42\left(2-C_{E}\right), 81.12\left(1-C_{E}\right), 80.44\left(2-C_{E}\right), 75.48\left(1-C_{Z}\right), 33.03\left(5-C_{E}\right), 31.84$ $\left(5-C_{Z}\right), 29.76$ (6-C, 7-C, 8-C, 9-C, 10-C), 29.45 (11-C), 29.22 (12-C), 29.10 (13-C), 22.62 (14C), 14.12 (15-C); IR (KBr) 3315, 3024, 2104, 1656, 962, 758, $616 \mathrm{~cm}^{-1}$.

\section{Acknowledgements}

We acknowledge the Estonian Science Foundation (grant No. 3368) for financial support.

\section{References}

1. Brown, C. A.; Yamashita, A. J. Am .Chem. Soc. 1975, 97, 891.

2. Brown, C. A. J. Chem. Soc.; Chem. Commun. 1975, 222.

3. Hommes, H.; Brandsma, L. Recl.Trav.Chim.Pays-Bas. 1977, 96, 160.

4. Abrams, S. R. Can. J .Chem. 1984, 62, 1333.

5. Abrams, S. R.; Nucciarone, D. D.; Steck, W. F. Can. J. Chem. 1983, 61, 1073.

6. Abrams, S. R.; Shaw, A. C. J. Org .Chem. 1987, 52, 1835.

7. Abrams, S. R. Can. J .Chem. 1982, 60, 1238.

8. Abrams, S. R. Can. J. Chem. 1986, 64,457.

9. Remizova, L. A.; Balova, I. A.; Favorskaja, I. A. J.Org.Chem.USSR 1986, 22, 2459.

10. Remizova, L. A.; Krukov, A. B.; Balova, I. A.; Favorskaja, I. A.; J.Org.Chem. USSR 1985, 21,1001 .

11. Brandsma, L. Preparative acetylenic chemistry; Elsevier: Amsterdam, 1971.

12. Mäeorg, U.; Talu, L.; Kallas, K. Proc.Estonian.Acad.Sci.Chem. 1996, 45, 140.

13. Brandsma, L.; Montijn, P. P.; Arens, J. F. Rec.trav.chim. 1963, 82, 1115.

14. Organikum, 12 Auflage, VEB Deutscher Verlag der Wissenschaften: Berlin, 1973. 